\title{
Propagation of Plasma
}

L-Phenylalanine Concentration Fluctuations to the Neurovascular Unit in Phenylketonuria: An in silico Study

\author{
Mehdi Taslimifar ${ }^{1,2}$, Stefano Buoso ${ }^{1,3}$, Francois Verrey $^{2,4,5 t}$ and Vartan Kurtcuoglu ${ }^{1,4,5,6 * t}$ \\ ${ }^{1}$ The Interface Group, Institute of Physiology, University of Zurich, Zurich, Switzerland, ${ }^{2}$ Epithelial Transport Group, Institute \\ of Physiology, University of Zurich, Zurich, Switzerland, ${ }^{3}$ Institute for Diagnostic and Interventional Radiology, Zurich \\ University Hospital, Zurich, Switzerland, ${ }^{4}$ Zurich Center for Integrative Human Physiology, University of Zurich, Zurich, \\ Switzerland, ${ }^{5}$ National Center of Competence in Research, Kidney Control of Homeostasis, Zurich, Switzerland, \\ ${ }^{6}$ Neuroscience Center Zurich, University of Zurich, Zurich, Switzerland
}

OPEN ACCESS

Edited by:

Guido Santos-Rosales, University Hospital Erlangen, Germany

Reviewed by:

Stefan Broer,

Australian National University,

Australia

Natalia Jarzebska,

Dresden University of Technology,

Germany

${ }^{*}$ Correspondence:

Vartan Kurtcuoglu

vartan.kurtcuoglu@uzh.ch

${ }^{\dagger}$ These authors have contributed equally to this work

Specialty section:

This article was submitted to Membrane Physiology

and Membrane Biophysics, a section of the journal

Frontiers in Physiology

Received: 23 October 2018 Accepted: 14 March 2019

Published: 02 April 2019

Citation:

Taslimifar M, Buoso S, Verrey F and Kurtcuoglu V (2019) Propagation

of Plasma L-Phenylalanine

Concentration Fluctuations to the

Neurovascular Unit

in Phenylketonuria: An in silico Study.

Front. Physiol. 10:360

doi: 10.3389/fphys.2019.00360
Phenylketonuria (PKU) is an inherited metabolic disease characterized by abnormally high concentrations of the essential amino acid L-phenylalanine (Phe) in blood plasma caused by reduced activity of phenylalanine hydroxylase (PAH). While numerous studies have shown association between high plasma Phe concentration and intellectual impairment, it is not clear whether increased Phe fluctuations also observed in PKU affect the brain as well. To investigate this, time-resolved in vivo data on Phe and competing large neutral amino acid (LNAA) concentrations in neurons are needed, but cannot be acquired readily with current methods. We have used in silico modeling as an alternative approach to characterize the interactive dynamics of Phe and competing LNAAs (CL) in the neurovascular unit (NVU). Our results suggest that plasma Phe fluctuations can propagate into the NVU cells and change there the concentration of LNAAs, with the highest magnitude of this effect observed at low frequency and high amplitude-to-mean ratio of the plasma Phe concentration fluctuations. Our model further elucidates the effect of therapeutic LNAA supplementation in PKU, showing how abnormal concentrations of Phe and CL in the NVU move thereby toward normal physiologic levels.

Keywords: phenylketonuria, L-phenylalanine fluctuation, neurovascular unit, amino acid transporter, large neutral amino acid

\section{INTRODUCTION}

Phenylketonuria (PKU) is the most common disorder of amino acid (AA) metabolism, resulting from severely reduced activity of the liver enzyme phenylalanine hydroxylase (PAH), which leads to abnormal accumulation of the essential amino acid L-phenylalanine (Phe) in the blood plasma (Güttler et al., 1969; Madden, 2004; Regier and Greene, 2017). Phe is transported across the blood brain barrier ( $\mathrm{BBB})$ into the neurovascular unit (NVU), where abnormal increase in its concentration leads to imbalance of large neutral amino acid (LNAA) levels in brain interstitial fluid (ISF), astrocytes and neurons, as a consequence of the competition for NVU-LNAA transporters (van Spronsen et al., 2010). Since NVU-LNAAs are required for the synthesis of 
essential neurotransmitters such as dopamine, serotonin, and norepinephrine, their perturbations can lead to intellectual disabilities, growth abnormalities and severe behavioral problems in PKU patients (Güttler et al., 1969; Cleary et al., 2013).

The dysfunction of the PAH enzyme is also the cause of higher than normal fluctuations in Phe plasma concentration, where patterns greatly vary among patients in relation to age, diet, genotype and level of PAH defect (Burgard et al., 1996; Cleary et al., 2013). Nevertheless, only time-averaged Phe concentrations are used to classify PKU severity (benign, mild or classic) and design the treatment protocol, which consists mainly of a low Phe diet to reduce mean blood Phe concentration (Cleary et al., 2013; Belanger et al., 2018). However, the effectiveness of such therapies depends on the concentrations of LNAAs in the NVU, which can influence brain function and behavior, rather than directly the blood Phe level (Cleary et al., 2013). Some studies have specifically investigated the relation between brain function (assessed through intelligence quotient (IQ) tests) and mean plasma Phe concentration and its fluctuations. The results were not conclusive: while in some studies IQ was found to be more often associated with the level of Phe fluctuations in the plasma rather than with its mean value (Hood et al., 2014; Romani et al., 2017), the opposite was reported in others (Viau et al., 2011; Hood et al., 2015), while no differentiation was possible in yet another one (Burgard et al., 1996). The reasons for this inconsistency are not clear, but could be, in part, a reflection of how fluctuations in plasma Phe concentration translate in individual patients to effects on AA homeostasis in the brain (Cleary et al., 2013).

The propagation of Phe fluctuations from plasma into the brain is critically influenced by the competition between this AA and its competing LNAAs (CL, i.e., L-leucine, L-isoleucine, L-tyrosine, L-tryptophan, L-valine, L-histidine, and L-methionine) for transporters at NVU cell membranes (Taslimifar et al., 2018). It has been shown that the $\mathrm{Na}^{+}$independent antiporter SLC7A5 (LAT1) in microvascular brain endothelial cells (MBEC) (Smith et al., 1987; Killian and Chikhale, 2001; Meier et al., 2002), the $\mathrm{Na}^{+}$-independent antiporter SLC7A8 (LAT2) in astrocytes (Yudkoff et al., 1996a; Kim et al., 2004; Braun et al., 2011), and the $\mathrm{Na}^{+}$-dependent symporter SLC6A15 (B ${ }^{0}$ AT2) in neurons (Yudkoff et al., 1996b; Bröer et al., 2006; Bak et al., 2012) are the main regulators of LNAAs homeostasis in the NVU (Figure 1). In vivo monitoring of LNAA levels in individual NVU compartments during fluctuations of plasma Phe concentrations could help establish a better understanding of their interrelation, but there are technological hurdles that need to be overcome to enable corresponding experiments.

To go around these hurdles, we have employed a previously developed computational model of NVU-LNAAs homeostasis (Taslimifar et al., 2018) to explore in silico how plasma Phe fluctuations influence LNAA concentrations in the NVU. In addition, we have quantified the variations in concentration of Phe and CL in NVU cells in relation to descriptors of plasma Phe fluctuation, namely mean, fundamental frequency and amplitude-to-mean ratio (Anastasoaie et al., 2008; Cleary et al., 2013). Finally, we have employed the model to explore the impact of therapeutic supplementation of LNAAs on the attenuation of

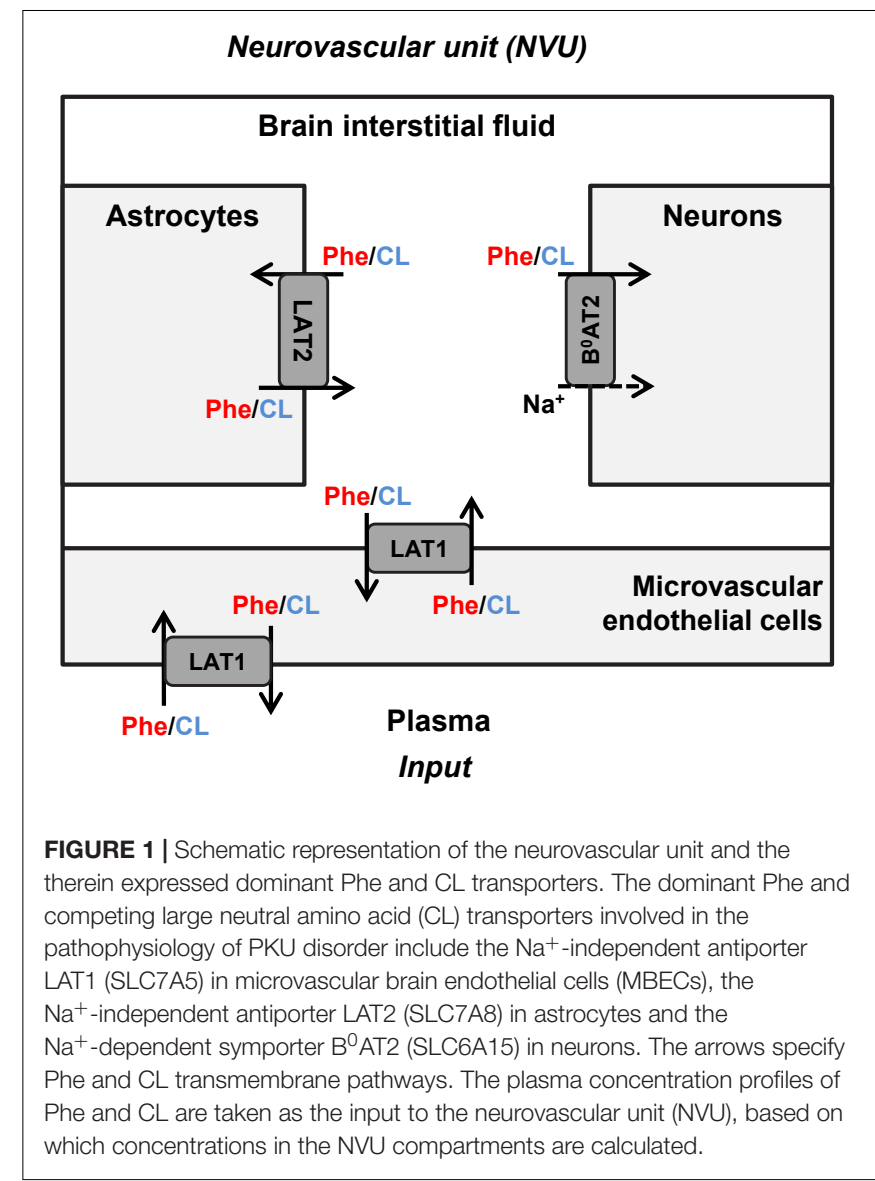

Phe and CL concentrations in NVU cells. While this treatment strategy has been shown to modulate the perturbed concentration of LNAAs in brain tissue in a PKU mouse model (van Vliet et al., $2015,2016)$ and also to positively impact executive functioning of PKU patients (Schindeler et al., 2007; van Spronsen et al., 2010), it remains unclear how supplementation of LNAAs affects the dynamics of Phe and CL concentration in MBECs, astrocyte and more importantly in neurons (van Spronsen et al., 2010; van Vliet et al., 2016).

\section{MATERIALS AND METHODS}

\section{Transport Model}

We employed a previously developed compartmental model of NVU-LNAA homeostasis in adult rats (Taslimifar et al., 2018), to which we refer the reader for detail. Four individual NVU compartments are considered - MBECs, ISF, astrocytes (ast), and neurons (neu) - within which LNAAs are assumed to be homogeneously distributed (Figure 1). In the model, LNAA fluxes between compartments are mediated by the dominant transporters identified from literature, i.e., LAT1, LAT2, and $\mathrm{B}^{0} \mathrm{AT} 2$ (Table 1). Fluxes mediated by the antiporters LAT1 and LAT2 are a function of the following: maximum transport rates at the BBB luminal (lum) ( $\left.\mathrm{V}_{\text {max,LAT1,lum }}\right)$ and abluminal (abl) ( $\left.\mathrm{V}_{\max , \mathrm{LAT} 1, \mathrm{abl}}\right)$ membranes and astrocyte 
TABLE 1 | Model parameters.

\begin{tabular}{|c|c|c|c|}
\hline & Value & Unit & Reference \\
\hline \multicolumn{4}{|l|}{ LAT1 (MBEC) } \\
\hline $\mathrm{K}_{\mathrm{m}, \mathrm{abs}, \mathrm{LAT} 1, \mathrm{Phe}}^{\mathrm{P} \text { ISF }}$ & 11 & $\mu \mathrm{M}$ & Smith et al., 1987 \\
\hline$V_{\max , \text { LAT1, lum, Phe }}$ & 0.075 & $\mu \mathrm{mol} / \mathrm{min}$ & $\begin{array}{l}\text { Smith et al., 1987; } \\
\text { Tilgmann et al., } 1992\end{array}$ \\
\hline $\mathrm{K}_{\mathrm{m}, \mathrm{abs}, \mathrm{LAT} 1, \mathrm{CL}}^{\mathrm{P}(\mathrm{SSF})}$ & $52.9^{*}$ & $\mu \mathrm{M}$ & Smith et al., 1987 \\
\hline $\mathrm{V}_{\max , \mathrm{LAT} 1, \mathrm{lum}, \mathrm{CL}}$ & $0.129 *$ & $\mu \mathrm{mol} / \mathrm{min}$ & $\begin{array}{l}\text { Smith et al., 1987; } \\
\text { Tilgmann et al., } 1992\end{array}$ \\
\hline RKLAT1 & 80 & - & Taslimifar et al., 2018 \\
\hline \multicolumn{4}{|c|}{ LAT2 (ASTROCYTE) } \\
\hline $\mathrm{K}_{\mathrm{m}, \mathrm{Ibs}, \mathrm{ISAT} \text { (Ast) Phe }}^{\mathrm{S}}$ & $110.2^{*}$ & $\mu \mathrm{M}$ & Kim et al., 2004 \\
\hline$V_{\max , L A T 2, P h e}$ & 0.1128 & $\mu \mathrm{mol} / \mathrm{min}$ & $\begin{array}{l}\text { Shank and Campbell, } \\
\text { 1984; Segawa et al., } 1999\end{array}$ \\
\hline $\mathrm{K}_{\mathrm{m}, \mathrm{abs}, \mathrm{ISF} \text { (Ast),CL }}$ & $185.9^{*}$ & $\mu \mathrm{M}$ & Kim et al., 2004 \\
\hline$V_{\max , L A T 2, C L}$ & $0.1494^{*}$ & $\mu \mathrm{mol} / \mathrm{min}$ & $\begin{array}{l}\text { Shank and Campbell, } \\
\text { 1984; Segawa et al., } 1999\end{array}$ \\
\hline \multicolumn{4}{|c|}{ B0AT2 (NEURON) } \\
\hline $\mathrm{K}_{\mathrm{m}, \mathrm{abs}, \mathrm{B}^{0} \mathrm{AT} 2 \text {,Phe }}^{\mathrm{ISF}(\mathrm{Neu})}$ & 1050 & $\mu \mathrm{M}$ & Bröer et al., 2006 \\
\hline $\mathrm{V}_{\max , \mathrm{B}^{0} \mathrm{AT} 2, \mathrm{Phe}}$ & $0.0086^{*}$ & $\mu \mathrm{mol} / \mathrm{min}$ & $\begin{array}{l}\text { Rao et al., 1995; Bröer } \\
\text { et al., } 2006\end{array}$ \\
\hline $\mathrm{K}_{\mathrm{m}, \mathrm{abs}, \mathrm{B}^{0} \mathrm{AT} 2, \mathrm{CL}}^{\mathrm{ISF}(\mathrm{Neu})}$ & $126.2^{*}$ & $\mu \mathrm{M}$ & Bröer et al., 2006 \\
\hline $\mathrm{V}_{\max , \mathrm{B}^{0} \mathrm{AT} 2, \mathrm{CL}}$ & $0.0186^{*}$ & $\mu \mathrm{mol} / \mathrm{min}$ & $\begin{array}{l}\text { Rao et al., 1995; Bröer } \\
\text { et al., } 2006\end{array}$ \\
\hline $\mathrm{K}_{\mathrm{m}, \mathrm{B}^{0} \mathrm{AT} 2, \mathrm{Na}}^{\mathrm{ISF}(\mathrm{Neu})}$ & 1050 & $\mu \mathrm{M}$ & Takanaga et al., 2005 \\
\hline$\Delta \Psi$ & -70 & $\mathrm{mV}$ & Smith et al., 1981 \\
\hline$\beta$ & $0.6^{*}$ & $\mathrm{mV}$ & $\begin{array}{l}\text { Takanaga et al., 2005; } \\
\text { Panitchob, } 2015\end{array}$ \\
\hline$[\mathrm{Na}]^{\mathrm{ISF}}$ & 141 & $\mathrm{mM}$ & Mori et al., 2002 \\
\hline$[\mathrm{Na}]^{\mathrm{Neu}}$ & 40 & $\mathrm{mM}$ & $\begin{array}{l}\text { Fedoroff and Vernadakis, } \\
1986\end{array}$ \\
\hline $\mathrm{V}_{\mathrm{MBEC}}$ & 3.5 & $\mu l$ & $\begin{array}{l}\text { Mori et al., 2002; Licinio } \\
\text { and Wong, } 2009\end{array}$ \\
\hline$V_{\text {ISF }}$ & 352.6 & $\mu l$ & $\begin{array}{l}\text { Tilgmann et al., 1992; } \\
\text { Syková et al., } 2005\end{array}$ \\
\hline $\mathrm{V}_{\text {Ast }}$ & 742 & $\mu l$ & $\begin{array}{l}\text { Ren et al., 1992; Anderova } \\
\text { et al., } 2011\end{array}$ \\
\hline $\mathrm{V}_{\mathrm{Neu}}$ & 441.7 & $\mu l$ & $\begin{array}{l}\text { Ren et al., 1992; Setou } \\
\text { et al., 2004; } \\
\text { Hosseini-Sharifabad and } \\
\text { Nyengaard, } 2007\end{array}$ \\
\hline
\end{tabular}

* The details of calculation processes are reported in Taslimifar et al. (2018).

membrane $\left(\mathrm{V}_{\max , \mathrm{LAT} 2}\right)$, respectively; Michaelis-Menten binding constants in the individual NVU compartments $\left(\mathrm{K}_{\mathrm{m}, \mathrm{LAT} 1}^{\mathrm{P}(\mathrm{MBEC}, \mathrm{ISF})}\right.$ and $\mathrm{K}_{\mathrm{m}, \mathrm{LAT} 2}^{\mathrm{ISF}(\text { Ast })}$; and the LAT1 bi-directional kinetic constant $\left(\mathrm{RK}_{\mathrm{LAT} 1}\right)$, which corresponds to the ratio between the absolute Michaelis-Menten binding constant for LAT1 in MBECs relative to the corresponding value in the ISF and in plasma $\left(\frac{\mathrm{K}_{\mathrm{m}, \mathrm{abs}, \mathrm{LAT1}}^{\mathrm{MBE}}}{\mathrm{K}_{\mathrm{m}, \mathrm{abs}, \mathrm{LAT1}}^{\mathrm{P}(\mathrm{LA})}}\right)$ (Taslimifar et al., 2018). The fluxes mediated by $\mathrm{B}^{0} \mathrm{AT} 2$ symporter are a function of Michaelis-Menten kinetic parameters $\left(\mathrm{V}_{\max , \mathrm{B}^{0} \mathrm{AT} 2}\right.$ and $\mathrm{K}_{\mathrm{m}, \mathrm{B}^{0} \mathrm{AT} 2}^{\mathrm{ISF}(\mathrm{Neu})}$; neuronal electrical potential-induced biases for forward and backward transport rates $\left(\varepsilon\right.$ and $\left.\varepsilon^{\prime}\right)$; neuronal potential difference $(\Delta \psi)$, electrical bias constant $(\beta)$, Faraday constant $(\mathrm{F})$, sodium charge $(\mathrm{Z})$, gas constant $(\mathrm{R})$, and absolute temperature (T) [see (Taslimifar et al., 2018) for details]. To account for the approximate 1:1 stoichiometry observed for LAT1 and LAT2 antiporters under normal physiologic conditions (Meier et al., 2002; Verrey, 2003), at each instant we limited Phe and CL fluxes to the lowest value between the two. The values of kinetic parameters and compartment volumes $\left(\mathrm{V}_{\mathrm{i}}\right)$ used in the model are taken from literature, and are reported in Table 1. The same values are considered for both PKU and normal physiologic cases (Möller et al., 1997; Taslimifar et al., 2018).

Model calculations were performed as follows: We first determined steady state (ss) concentrations of Phe and CL in the individual NVU compartments $i$ ([Phe $]_{s s}^{\mathrm{i}}$ and $\left.[\mathrm{CL}]_{\mathrm{SS}}^{\mathrm{i}}\right)$ by prescribing constant plasma concentrations ([Phe $]_{\mathrm{ss}}^{\mathrm{P}}$ and $[\mathrm{CL}]_{\mathrm{ss}}^{\mathrm{P}}$ ) as model inputs and initializing the concentrations in the NVU compartments based on steady state concentration values reported in Taslimifar et al. (2018). We considered plasma Phe concentrations from $77 \mu \mathrm{M}$ (representing normal physiologic condition) (Currie et al., 1995) to values above $1200 \mu \mathrm{M}$ (representing sever classic PKU) (Güttler et al., 1969; Regier and Greene, 2017). Plasma CL concentration was kept constant at 739 нM (Currie et al., 1995; Bongiovanni et al., 2003).

We then prescribed fluctuating plasma Phe concentrations as

$$
[\mathrm{Phe}]_{\mathrm{f}}^{\mathrm{P}}=[\mathrm{Phe}]_{\mathrm{ss}}^{\mathrm{P}}\left(1+\mathrm{c}_{\mathrm{f}} \frac{[\mathrm{Phe}]_{\delta}^{\mathrm{P}}}{\max \left|[\mathrm{Phe}]_{\delta}^{\mathrm{P}}\right|}\right)
$$

where $c_{\mathrm{f}}$ is a coefficient that can take on values between 0 and 1 , and thereby scales the fluctuation amplitude (relative to mean), and $[\mathrm{Phe}]_{\delta}^{\mathrm{P}}$ is a periodic zero-mean fluctuating component with zero initial value described by the trigonometric Fourier series

$$
[\text { Phe }]_{\delta}^{\mathrm{P}}=\sum_{\mathrm{n}=1}^{\mathrm{N}} \mathrm{a}_{\mathrm{n}} \sin \left(2 \pi \mathrm{nf}_{0} \mathrm{t}\right)+b_{\mathrm{n}} \cos \left(2 \pi \mathrm{nf}_{0} \mathrm{t}\right),
$$

where $n$ is an integer, $f_{o}$ is the fundamental frequency of Phe fluctuation, and $a_{n}$ and $b_{n}$ are the Fourier coefficients.

For presentation purposes, we initially considered three distinct plasma Phe fluctuation profiles referred to as cases c1c3, with values of steady state concentration, frequency and relative amplitude as given in Table 2 . For each of these cases, we determined the steady state concentrations of Phe and $\mathrm{CL}\left([\mathrm{Phe}]_{\mathrm{ss}}^{\mathrm{i}}\right.$ and $\left.[\mathrm{CL}]_{\mathrm{ss}}^{\mathrm{i}}\right)$ as well as the fluctuating response $\left([\mathrm{Phe}]_{\mathrm{f}}^{\mathrm{i}}\right.$ and $[\mathrm{CL}]_{\mathrm{f}}^{\mathrm{i}}$ ) in the NVU compartments. Results for this in silico investigation are shown as time series (excluding the initial transition from the steady state) normalized with respect to the corresponding values for normal physiologic conditions reported in Table 3 (determined for model input $[\mathrm{Phe}]_{\mathrm{ss}}^{\mathrm{P}}=77 \mu \mathrm{M},[\mathrm{CL}]_{\mathrm{ss}}^{\mathrm{P}}=739 \mu \mathrm{M}$ and $\mathrm{c}_{\mathrm{f}}=0$; Currie et al., 1995; Bongiovanni et al., 2003).

To further investigate the effect of fluctuations, we considered a PKU case for which we fixed the non-fluctuating plasma Phe and CL concentrations and varied the fluctuation indices (fundamental frequency and amplitude-to-mean ratio) of a purely sinusoidal signal oscillation, $[\mathrm{Phe}]_{\delta}^{\mathrm{P}}=\sin \left(2 \pi \mathrm{f}_{0} \mathrm{t}\right)$, within realistic bounds $\left(0.14 \leq \mathrm{f}_{0} \leq 7\right.$ cycles per day and $\left.0 \leq \mathrm{c}_{\mathrm{f}} \leq 1\right)$ 
TABLE 2 | Fluctuation indices of exemplary Phe plasma concentration profiles.

\begin{tabular}{lccl}
\hline Case number & {$[\mathrm{Phe}]_{\mathbf{s s}}^{\mathbf{P}}(\boldsymbol{\mu} \mathbf{M})$} & $\mathbf{f}_{\mathbf{0}}$ (cycles/day) & $\mathbf{c}_{\mathbf{f}}(-)$ \\
\hline c1 & 300 & 1 & 0.99 \\
c2 & 800 & 3 & 0.3 \\
c3 & 1600 & 0.14 & 0.6 \\
\hline
\end{tabular}

TABLE 3 | Steady state normal physiologic (baseline) concentration values of Phe and $\mathrm{CL}$ in the NVU.

\begin{tabular}{lccc}
\hline Compartment & Parameter & Concentration & Unit \\
\hline Microvascular brain & {$[\mathrm{Phe}]_{\mathrm{SS}}^{\mathrm{MBEC}}$} & $24.6 \pm 146.8$ & $\mu \mathrm{M}$ \\
endothelial cell & {$[\mathrm{CL}]_{\mathrm{SS}}^{\mathrm{MBEC}}$} & $235.7 \pm 1408.8$ & $\mu \mathrm{M}$ \\
& {$[\mathrm{Phe}]_{\mathrm{SS}}^{\mathrm{SF}}$} & $0.03 \pm 0.4$ & $\mu \mathrm{M}$ \\
Brain interstitial fluid & {$[\mathrm{CL}]_{\mathrm{SS}}^{\mathrm{SF}}$} & $0.3 \pm 3.8$ & $\mu \mathrm{M}$ \\
& {$[\mathrm{Phe}]_{\mathrm{SS}}^{\mathrm{Ast}}$} & $4.8 \pm 40.6$ & $\mu \mathrm{M}$ \\
Astrocyte & {$[\mathrm{CL}]_{S \mathrm{~S}}^{\mathrm{Ast}}$} & $46.0 \pm 389.7$ & $\mu \mathrm{M}$ \\
& {$[\mathrm{Phe}]_{\mathrm{SS}}^{\mathrm{Neu}}$} & $7.0 \pm 53.4$ & $\mu \mathrm{M}$ \\
Neuron & {$[\mathrm{CL}]_{\mathrm{SS}}^{\mathrm{Neu}}$} & $46.9 \pm 368.7$ & $\mu \mathrm{M}$ \\
& & & \\
\hline
\end{tabular}

The details for the calculation of the steady state conditions are reported in Taslimifar et al. (2018). The plasma concentrations of Phe and CL are 77 and $739 \mu \mathrm{M}$, respectively (Currie et al., 1995; Bongiovanni et al., 2003).

(Güttler et al., 1969; MacDonald et al., 1998; Ferguson and Morris, 1999; Michals-Matalon et al., 2007; Mitchell et al., 2011). We, similarly, calculated the fluctuating and steady state concentrations of Phe and CL in the NVU compartments. For each compartment, we calculated the root mean square of the difference between the fluctuating and steady state responses (normalized with respect to normal physiologic conditions) and considered it as a metric for the excursion of the concentration of Phe and CL.

Finally, we employed the model to investigate the impact of therapeutic supplementation of non-Phe LNAAs on the concentrations of Phe and CL in NVU cells. To this end, we considered the supplemented LNAAs (SL) as CL with the same kinetics, and prescribed it as constant input (representing the effective plasma concentration of SL) to the model. We then determined steady state and fluctuating responses in the NVU, considering, as model input, different values for the effective steady state concentrations of SL in the plasma $(0.5$, 2 , and $5 \mathrm{mM})$ and of both steady state $\left([\mathrm{Phe}]_{\mathrm{ss}}^{\mathrm{P}}=1000\right.$ and $\left.\mathrm{c}_{\mathrm{f}}=0\right)$ and sinusoidally fluctuating plasma Phe concentration $\left([\mathrm{Phe}]_{\delta}^{\mathrm{P}}=\sin \left(2 \pi \mathrm{f}_{0} \mathrm{t}\right),[\mathrm{Phe}]_{\mathrm{ss}}^{\mathrm{P}}=1000 \mu \mathrm{M}, \mathrm{c}_{\mathrm{f}}=0.5\right.$ and $\mathrm{f}_{0}=1$ cycles/day). We show the responses as time series (excluding transition from steady state) normalized with respect to the corresponding values of baseline physiologic conditions.

\section{Sensitivity Analysis}

We evaluated the sensitivity of the reported results on the choice of literature-reported model parameter values. To this end, we calculated model output for 100 cases in which the nominal model input parameters [maximum transport rates, Michaelis-Menten binding constants and steady state physiologic concentration values of LNAAs in individual compartments (Tables 1, 3)] were varied randomly in a range of $\pm 20 \%$ from their respective nominal values. In the results, the shown upper and lower bounding curves correspond to standard deviations of the calculated concentrations from those computed under nominal parameter conditions.

\section{RESULTS}

\section{NVU Steady State Condition in Relation to Steady State Plasma Phe Concentration}

Figure 2 shows plots of steady state concentrations of Phe and CL in neurons versus steady state concentrations of plasma Phe from $77 \mu \mathrm{M}$ (normal physiologic conditions) to values above $1200 \mu \mathrm{M}$ (severe classic PKU). The reason why AA levels in the modeled neuron reach equilibrium (even though the $\mathrm{Na}^{+}$ electrochemical force drives influx through $\mathrm{B}^{0} \mathrm{AT} 2$ ) is their low extracellular concentration in the brain interstitial fluid. Phe and CL concentrations in the other compartments are shown in Supplementary Figure S1. In all NVU compartments, Phe concentration increases with its plasma concentration, while the opposite behavior is observed for CL. In Supplementary Figure S1E, we show the steady state responses in the whole brain tissue as volume-weighted average of the steady state responses in the individual NVU compartments. Given that animal species are different in terms of NVU geometry and also kinetic characteristics of LNAA transporters (Smith et al., 1987; Hargreaves and Pardridge, 1988; Martynyuk et al., 2010), a 1:1 comparison between species cannot be provided for concentration behavior of LNAA in the brain. However, the observed growth trend we determined for Phe in whole brain tissue in our in silico adult PKU rat brain model is qualitatively in line with experimental observations in a PKU mouse model (van Vliet et al., 2015; Belanger et al., 2018).

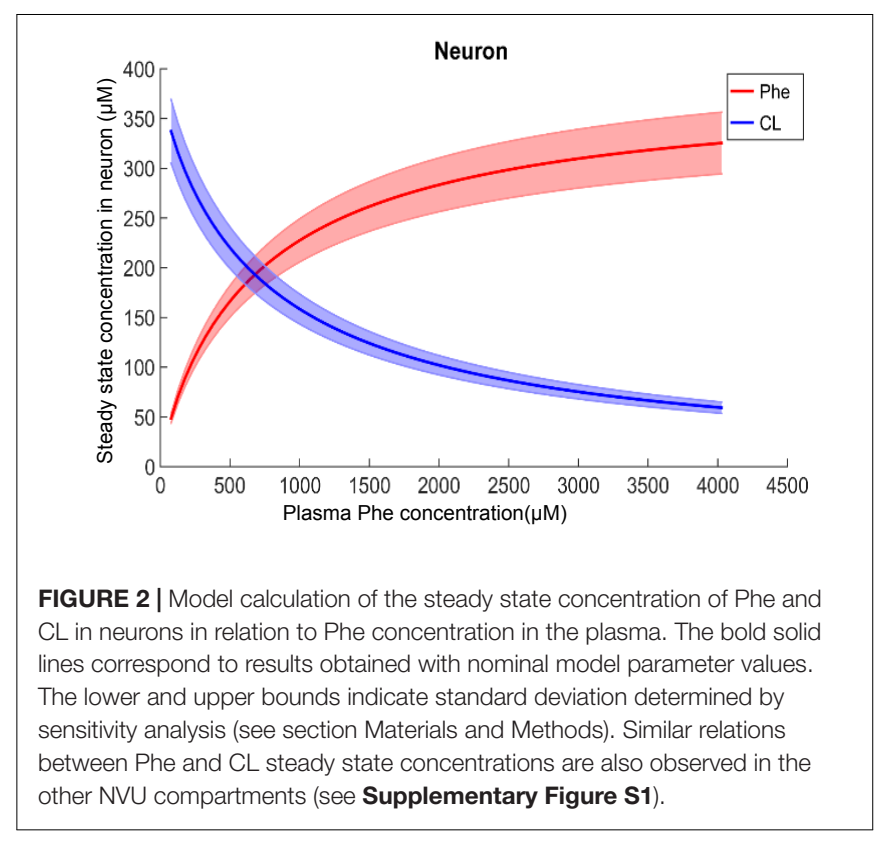




\section{The Dynamics of LNAAs in the NVU Are Influenced by Phe Concentration Fluctuations in the Plasma}

After the assessment of the NVU steady state response to steady state plasma Phe concentrations, we calculated the dynamic changes of Phe and CL in the NVU in response to plasma Phe fluctuations. Figure 3 shows the evolution of the concentrations of Phe and CL over time in MBECs (Figures 3B1-B3), brain ISF (Figures 3C1-C3), astrocytes (Figures 3D1-D3), and neurons (Figures 3E1-E3) for the three plasma Phe fluctuation cases c1, c2, and c3 (Figures 3A1-A3) (normalized with respect to normal physiologic conditions) over a span of 14 days. The shown upper and lower bounding curves correspond to standard deviations obtained through sensitivity analysis (see section Materials and Methods). In all cases, the results highlight the competition between Phe and CL: for example, the transfer of Phe through MBEC via the antiporter LAT1 is associated with movements of $\mathrm{CL}$ in reverse direction at both luminal and abluminal BBB membranes (Figures 3B1-B3). Once Phe and CL gain entry into the brain ISF (Figures $3 \mathrm{Cl}-\mathrm{C} 3$ ), they are either exchanged back into MBECs through abluminal LAT1 (Figures 3B1-B3) and/or astrocytes via LAT2 (Figures 3D1D3), and/or co-transported along with sodium ions into neurons via $\mathrm{B}^{0} \mathrm{AT} 2$ (Figures 3E1-E3). Additionally, in all cases, the steady state Phe concentration in brain ISF, astrocyte and neurons are characterized by values higher than under normal physiologic conditions (which correspond to "baseline" or $100 \%$ in the figure), while the opposite is observed for CL concentrations. We also observe that the concentration dynamics of both Phe and CL in the NVU compartments are influenced by plasma Phe fluctuations, with the impact dependent on plasma Phe fluctuation frequency, mean value, and amplitude-to-mean ratio. For example, in case c1, plasma Phe fluctuations strongly affect the dynamics of Phe and CL concentration in brain ISF and MBECs and, to a lesser extent, in astrocytes and neurons.

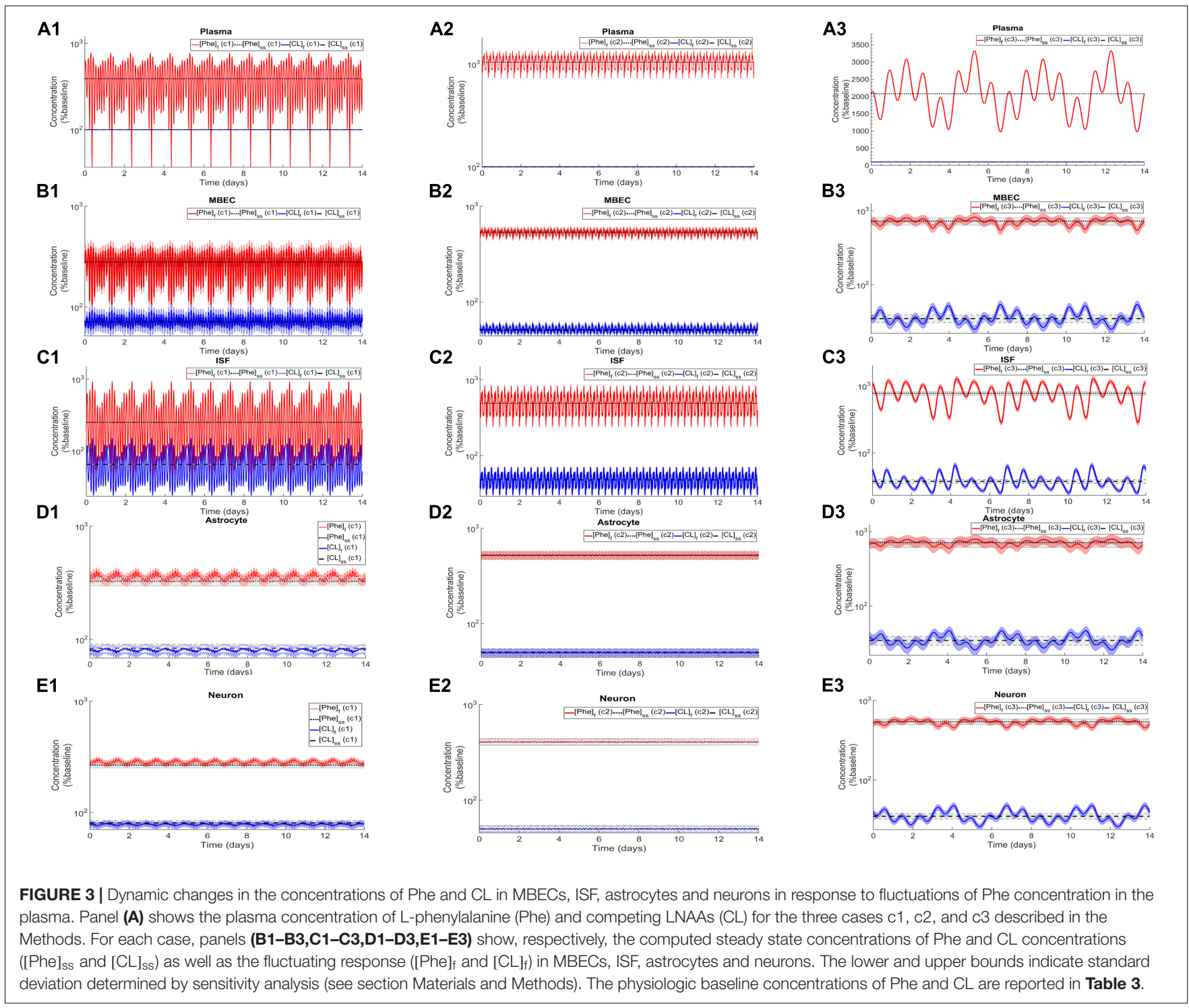


In case $\mathrm{c} 2$, the variations in concentrations of Phe and $\mathrm{CL}$ in astrocyte and neurons are not significant as compared to corresponding changes in MBECs and brain ISF. Finally, in case c3, we observe large variations in concentrations of Phe and $\mathrm{CL}$ in brain ISF and MBEC, but comparably lower variations in astrocytes and neurons.

\section{Association Between Plasma Phe Fluctuation and Changes in Phe and CL Concentration in the NVU}

To study how perturbations in Phe and CL concentrations in the NVU relate to plasma Phe fluctuation indices, we simultaneously varied $\mathrm{f}_{0}$ and $\mathrm{c}_{\mathrm{f}}$ of sinusoidally fluctuating plasma Phe concentration, and then calculated the corresponding changes in concentration of Phe and CL in the NVU compartments. We thereby quantified the associations between Phe and CL excursion and the plasma Phe frequency and amplitude-to-mean ratio. Supplementary Figure S2 shows corresponding results observed over a span of 14 days $\left([\mathrm{Phe}]_{\delta}^{\mathrm{P}}=\sin \left(2 \pi \mathrm{f}_{0} \mathrm{t}\right)\right.$ and $\left.[\mathrm{Phe}]_{\mathrm{SS}}^{\mathrm{P}}=1600 \mu \mathrm{M}\right)$. The largest Phe and CL changes are observed in all NVU compartments for large amplitude-to-mean ratio of Phe fluctuations in the plasma. Additionally, low frequencies lead to higher excursion for Phe and CL in astrocytes and neurons and also in ISF for CL, whereas in the same ISF compartment the
Phe excursions are lower under low frequencies (Supplementary Figures S2B1,2,C1,2,D1,2). Finally, we observed that in MBECs, excursion levels are not influenced by the fluctuation frequency and that the changes in Phe and CL concentration are also not significantly sensitive to fluctuation frequency in the other compartments when the fluctuation coefficient is small (Supplementary Figures S2A1-D2).

\section{Impact of LNAA Supplementation on Phe and CL Concentrations in the NVU}

To elucidate the impact of non-Phe LNAA supplementation, we determined Phe and CL concentrations in neurons (Figures 4B1,B2), MBECs (Supplementary Figures S3B1,B2), ISF (Supplementary Figures S3C1,C2) and astrocytes (Supplementary Figures S3D1,D2) in response to various concentrations of SL in the plasma under both steady state and fluctuating Phe conditions (Figures 4A1,A2). The shown upper and lower bounding curves correspond to standard deviations investigated by performing sensitivity analysis. Our results show that supplementation of LNAA attenuates the disturbed steady state concentration of Phe and CL in the NVU and modulates their concentrations toward physiologic baseline conditions (Table 3). This is because SL together with CL increases the competitions between Phe and non-Phe LNAAs through LAT1 across the BBB and thereby inhibits

\section{A1}

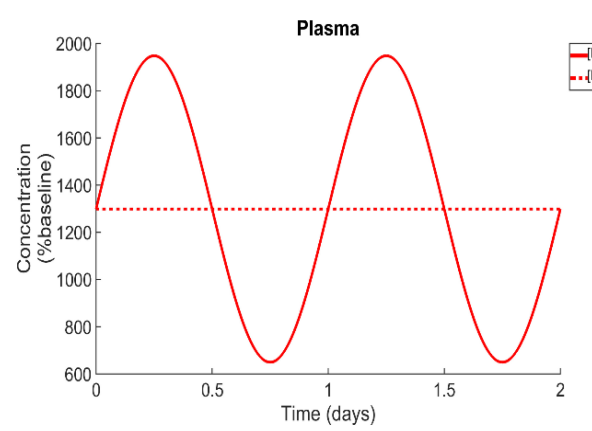

B1

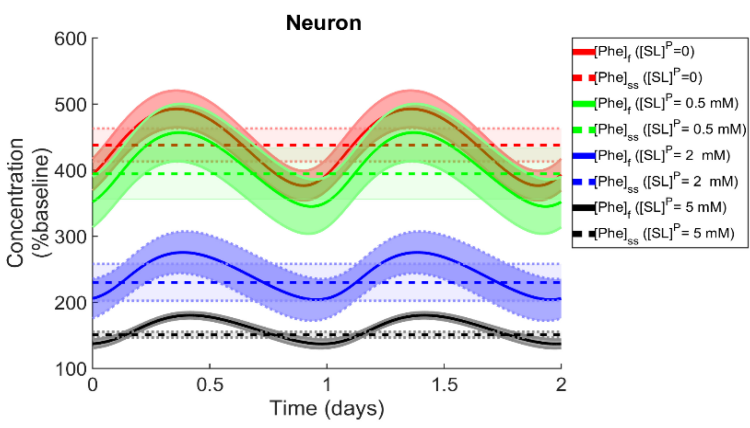

A2

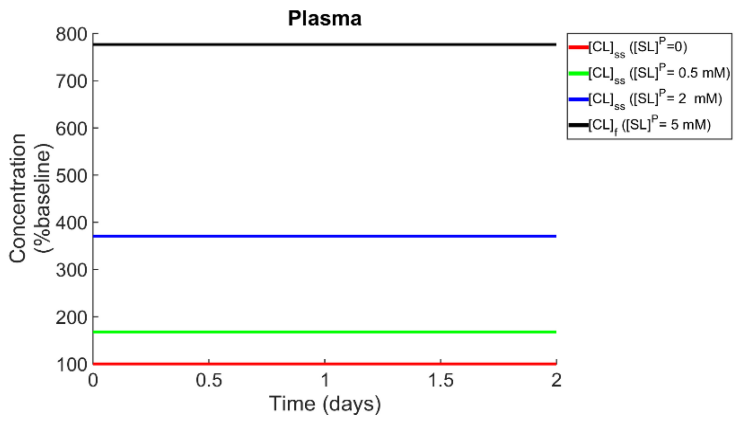

B2

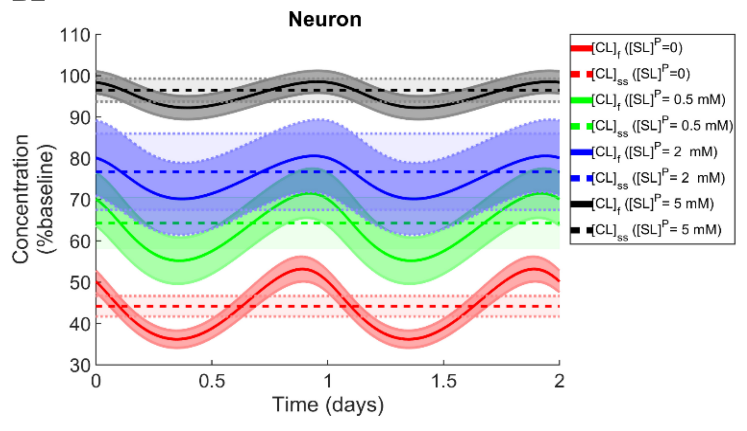

FIGURE 4 | Impact of LNAA supplementation on Phe and CL concentrations in the NVU. Panels (A1,A2) show the plasma concentration of L-phenylalanine (Phe) and competing LNAAs (CL) used as model input. Panels (B1,B2) show the model calculations for steady state concentrations of Phe and CL ([Phe $]_{s s}$ and $\left.[C L]_{s s}\right)$ as well as the fluctuating response ( $[\mathrm{Phe}]_{f}$ and $\left.[\mathrm{CL}]_{f}\right)$ in the neuronal compartment for various concentration levels of supplemented $\mathrm{LNAAs}$ in the plasma $\left([S L]^{\mathrm{P}}\right)$. The lower and upper bounds indicate standard deviation determined by sensitivity analysis (see section Materials and Methods). In all panels, the baseline physiologic concentrations of Phe and CL are reported in Table 3. 
LAT1 mediated trans-endothelial transport of Phe fluctuations from plasma into the brain. As a consequence, this leads to a reduction in the amplitude level of Phe and CL fluctuations in neurons, MBECs, ISF and astrocytes (Figures 4B1,B2 and Supplementary Figures S3B1,B2,C1,C2,D1,D2, respectively). In addition, the increased competition via LAT1 leads to delayed response in neurons, ISF and astrocytes (Figures 4B1,B2 and Supplementary Figures S3C1,C2,D1,D2, respectively).

\section{DISCUSSION}

Failure of AA homeostasis in general negatively affects cell function (Suraweera et al., 2012). In cells of the NVU, Phe and CL are involved in a variety of processes, including the synthesis of essential neurotransmitters such as dopamine (3-hydroxytyramine) and serotonin (5-hydroxytryptamine; 5HT) (Fernstrom, 1994, 2005; Lieberman, 2000; Cansev and Wurtman, 2007), and the production of proteins and lipids (e.g., myelin) (Sperringer et al., 2017; Yudkoff, 2017), although their function in the latter processes is not fully understood. Hence, depending on the full set of roles of Phe and CL, oscillations of their concentrations in the NVU could have a negative impact on central nervous system function. However, fluctuating NVU-LNAA concentrations are difficult to measure in animal models of PKU. Furthermore, in patient studies, the effects of elevated steady state plasma Phe concentration cannot be easily differentiated from those caused by increased fluctuations in Phe. The here introduced in silico model can serve as an alternative tool to probe these complex dynamics quickly and quantitatively.

We first showed that with increasing Phe concentration in plasma, the steady state concentration of Phe in the NVU and in whole brain increases, while CL concentration decreases. The growth trend we determined for Phe in our in silico adult PKU rat brain model is qualitatively in line with experimental observations in a PKU mouse model (van Vliet et al., 2015). We then showed that the propagation of plasma Phe concentration oscillations into the NVU largely depend on fluctuation indices such as frequency and amplitude-to-mean ratio. We observed the largest Phe and CL concentration excursions in the NVU for low frequencies and large amplitude-to-mean ratios of plasma Phe fluctuations. Additionally, we observed that, for the small fluctuation coefficients, the sensitivity of Phe and CL excursions to plasma Phe fluctuation frequency is not significant. Finally, we showed that supplementation of LNAAs not only reduces steady state concentrations in the NVU toward normal physiologic values, but also leads to a reduction in Phe and CL fluctuation amplitude. In conclusion, our in silico experiments support LNAA supplementation as therapeutic strategy for reducing the propagation of plasma Phe concentration fluctuations into the brain. The model itself can also be seen as a potential stepping-stone for the development of a patient-specific LNAA supplementation treatment-planning tool.

The computational model has been built with a number of simplifying assumptions. In particular, we focused on the pathways mediated by dominant transporters and thus disregarded pathways related to metabolism, diffusion and NVU transporters with low levels of expression, which have been shown to be of lesser importance (Sadasivudu and Lajtha, 1970; Smith and Takasato, 1986). Additionally, we considered Phe and $\mathrm{CL}$ as a homogenous mixture within the individual NVU compartments. Furthermore, we considered CL as a single entity rather than accounting one by one for each individual competitor, i.e., for L-isoleucine, L-tyrosine and others. The validity of these assumptions are discussed in more detail in Taslimifar et al. (2018). To account for effects of uncertainties associated with the values of the model parameters, we have performed a sensitivity analysis demonstrating that our conclusions are robust with respect to reasonable parameter variations.

\section{SUMMARY}

Using a computational model of adult rat NVU-LNAA homeostasis, we investigated the effects of plasma Phe fluctuations on the dynamics of LNAAs in MBECs, brain ISF, astrocytes and neurons in PKU. Comparable in vivo investigations are not readily doable with current techniques. Our results show that plasma Phe fluctuations can propagate into the NVU and change there the concentration of LNAAs, with the magnitude of this effect largely dependent on the frequency and amplitude-to-mean ratio of the plasma concentration fluctuations. Finally, we quantified the therapeutic impact of supplementation of LNAAs in attenuating the disturbed fluctuating and steady state concentrations of Phe and CL in the cells of NVU in PKU disorder toward normal physiologic levels.

\section{AUTHOR CONTRIBUTIONS}

MT implemented the computational model and performed the calculations with SB. FV and VK directed the research. All authors conceived and designed the study, analyzed the data, wrote the manuscript, and approved the final version.

\section{FUNDING}

This work was financially supported by SystemsX.ch - the Swiss Initiative in Systems Biology through the QuantX project, and by the Swiss National Science Foundation through NCCR Kidney.CH and grant 310030_166430.

\section{ACKNOWLEDGMENTS}

We would like to thank Nenad Blau for helpful discussions on the fluctuation of Phe concentration in blood.

\section{SUPPLEMENTARY MATERIAL}

The Supplementary Material for this article can be found online at: https://www.frontiersin.org/articles/10.3389/fphys.2019. 00360/full\#supplementary-material 


\section{REFERENCES}

Anastasoaie, V., Kurzius, L., Forbes, P., and Waisbren, S. (2008). Stability of blood phenylalanine levels and IQ in children with phenylketonuria. Mol. Genet. Metab. 95, 17-20. doi: 10.1016/j.ymgme.2008.06.014

Anderova, M., Vorisek, I., Pivonkova, H., Benesova, J., Vargova, L., Cicanic, M., et al. (2011). Cell death/proliferation and alterations in glial morphology contribute to changes in diffusivity in the rat hippocampus after hypoxiaischemia. J. Cereb. Blood Flow Metab. 31, 894-907. doi: 10.1038/jcbfm. 2010.168

Bak, L. K., Johansen, M. L., Schousboe, A., and Waagepetersen, H. S. (2012). Valine but not leucine or isoleucine supports neurotransmitter glutamate synthesis during synaptic activity in cultured cerebellar neurons. J. Neurosci. Res. 90, 1768-1775. doi: 10.1002/jnr.23072

Belanger, A. M., Przybylska, M., Gefteas, E., Furgerson, M., Geller, S., Kloss, A., et al. (2018). Inhibiting neutral amino acid transport for the treatment of phenylketonuria. JCI Insight 3:121762. doi: 10.1172/jci.insight.121762

Bongiovanni, R., Yamamoto, B. K., Simpson, C., and Jaskiw, G. E. (2003). Pharmacokinetics of systemically administered tyrosine: a comparison of serum, brain tissue and in vivo microdialysate levels in the rat. J. Neurochem. 87, 310-317. doi: 10.1046/j.1471-4159.2003.02007.x

Braun, D., Kinne, A., Bräuer, A. U., Sapin, R., Klein, M. O., Köhrle, J., et al. (2011). Developmental and cell type-specific expression of thyroid hormone transporters in the mouse brain and in primary brain cells. Glia 59, 463-471. doi: 10.1002/glia.21116

Bröer, A., Tietze, N., Kowalczuk, S., Chubb, S., Munzinger, M., Bak, L. K., et al. (2006). The orphan transporter v7-3 (slc6a15) is a Na+-dependent neutral amino acid transporter (B0AT2). Biochem. J. 393, 421-430. doi: 10.1042/ BJ20051273

Burgard, P., Rupp, A., Konecki, D., Trefz, F., Schmidt, H., and LichterKonecki, U. (1996). Phenylalanine hydroxylase genotypes, predicted residual enzyme activity and phenotypic parameters of diagnosis and treatment of phenylketonuria. Eur. J. Pediatr. 155, S11-S15. doi: 10.1007/PL00014222

Cansev, M., and Wurtman, R. (2007). "4 aromatic amino acids in the brain," in Handbook of Neurochemistry and Molecular Neurobiology, eds A. Lajtha, S. S. Oja, P. Saransaari, and A. Schousboe (Berlin: Springer), 59-97. doi: 10.1007/ 978-0-387-30373-4_4

Cleary, M., Trefz, F., Muntau, A. C., Feillet, F., van Spronsen, F. J., Burlina, A., et al. (2013). Fluctuations in phenylalanine concentrations in phenylketonuria: a review of possible relationships with outcomes. Mol. Genet. Metabol. 110, 418-423. doi: 10.1016/j.ymgme.2013.09.001

Currie, P. J., Chang, N., Luo, S., and Anderson, G. H. (1995). Microdialysis as a tool to measure dietary and regional effects on the complete profile of extracellular amino acids in the hypothalamus of rats. Life Sci. 57, 1911-1923. doi: 10.1016/0024-3205(95)02178-L

Fedoroff, S., and Vernadakis, A. (1986). Astrocytes: Biochemistry, Physiology, and Pharmacology of Astrocytes, Vol. 2. Orlando: Academic Press.

Ferguson, C., and Morris, A. (1999). Changes in serum phenylalanine after overnight fasts in youngsters with phenylketonuria. J. Hum. Nutr. Dietet. 12, 213-218. doi: 10.1046/j.1365-277x.1999.00158.x

Fernstrom, J. D. (1994). Dietary amino acids and brain function. J. Am. Dietet. Assoc. 94, 71-77. doi: 10.1016/0002-8223(94)92045-1

Fernstrom, J. D. (2005). Branched-chain amino acids and brain function. J. Nutr. 135, 1539S-1546S. doi: 10.1093/jn/135.6.1539S

Güttler, F., Olesen, E. S., and Wamberg, E. (1969). Diurnal variations of serum phenylalanine in phenylketonuric children on low phenylalanine diet. Am. J. Clin. Nutr. 22, 1568-1570. doi: 10.1093/ajcn/22.12.1568

Hargreaves, K., and Pardridge, W. (1988). Neutral amino acid transport at the human blood-brain barrier. J. Biol. Chem. 263, 19392-19397.

Hood, A., Antenor-Dorsey, J. A. V., Rutlin, J., Hershey, T., Shimony, J. S., McKinstry, R. C., et al. (2015). Prolonged exposure to high and variable phenylalanine levels over the lifetime predicts brain white matter integrity in children with phenylketonuria. Mol. Genet. Metab. 114, 19-24. doi: 10.1016/j. ymgme.2014.11.007

Hood, A., Grange, D. K., Christ, S. E., Steiner, R., and White, D. A. (2014). Variability in phenylalanine control predicts IQ and executive abilities in children with phenylketonuria. Mol. Genet. Metabol. 111, 445-451. doi: 10. 1016/j.ymgme.2014.01.012
Hosseini-Sharifabad, M., and Nyengaard, J. R. (2007). Design-based estimation of neuronal number and individual neuronal volume in the rat hippocampus. J. Neurosci. Methods 162, 206-214. doi: 10.1016/j.jneumeth.2007. 01.009

Killian, D. M., and Chikhale, P. J. (2001). Predominant functional activity of the large, neutral amino acid transporter (LAT1) isoform at the cerebrovasculature. Neurosci. Lett. 306, 1-4. doi: 10.1016/S0304-3940(01)01810-9

Kim, D. K., Kim, I. J., Hwang, S., Kook, J. H., Lee, M. C., Shin, B. A., et al. (2004). System L-amino acid transporters are differently expressed in rat astrocyte and C6 glioma cells. Neurosci. Res. 50, 437-446. doi: 10.1016/j.neures.2004.08.003

Licinio, J., and Wong, M.-L. (2009). Pharmacogenomics: The Search for Individualized Therapies. Hoboken: John Wiley \& Sons.

Lieberman, H. R. (2000). Amino Acid and Protein Requirements: Cognitive Performance, Stress, and Brain Function. Natick MA: Army Research Inst Of Environmental Medicine.

MacDonald, A., Rylance, G., Asplin, D., Hall, S., and Booth, I. (1998). Does a single plasma phenylalanine predict quality of control in phenylketonuria? Arch. Dis. Child. 78, 122-126. doi: 10.1136/adc.78.2.122

Madden, M. (2004). Phenylketonuria: defects in amino acid metabolism. Mol. Med. $5,57-61$.

Martynyuk, A., Van Spronsen, F., and Van der Zee, E. (2010). Animal models of brain dysfunction in phenylketonuria. Mol. Genet. Metab. 99, S100-S105. doi: 10.1016/j.ymgme.2009.10.181

Meier, C., Ristic, Z., Klauser, S., and Verrey, F. (2002). Activation of system L heterodimeric amino acid exchangers by intracellular substrates. EMBO J. 21, 580-589. doi: 10.1093/emboj/21.4.580

Michals-Matalon, K., Bhatia, G., Guttler, F., Tyring, S. K., and Matalon, R. (2007). Response of phenylketonuria to tetrahydrobiopterin. J. Nutr. 137, 1564S-1567S. doi: $10.1093 /$ jn/137.6.1564S

Mitchell, J. J., Trakadis, Y. J., and Scriver, C. R. (2011). Phenylalanine hydroxylase deficiency. Genet. Med. 13:697. doi: 10.1097/GIM.0b013e3182141b48

Möller, H. E., Weglage, J., Wiedermann, D., Vermathen, P., Bick, U., and Ullrich, K. (1997). Kinetics of phenylalanine transport at the human blood-brain barrier investigated in vivo. Brain Res. 778, 329-337. doi: 10.1016/S0006-8993(97) 01054-8

Mori, K., Miyazaki, M., Iwase, H., and Maeda, M. (2002). Temporal profile of changes in brain tissue extracellular space and extracellular ion $(\mathrm{Na}+$, $\mathrm{K}+$ ) concentrations after cerebral ischemia and the effects of mild cerebral hypothermia. J. Neurotrauma 19, 1261-1270. doi: 10.1089/089771502603 38047

Panitchob, N. (2015). Computational Modelling of Amino Acid Transfer Interactions in the Placenta. Doctoral thesis, University of Southampton, Southampton.

Rao, K. R., Vemuri, M. C., and Murthy, C. R. (1995). Synaptosomal transport of branched chain amino acids in young, adult and aged rat brain cortex. Neurosci. Lett. 184, 137-140. doi: 10.1016/0304-3940(94) 11189-P

Regier, D. S., and Greene, C. L. (2017). "Phenylalanine hydroxylase deficiency," in GeneReviews ${ }^{\circledR}$ [Internet], eds M. P. Adam, H. H. Ardinger, R. A. Pagon, et al. (Seattle: University of Washington). Available at: https://www.ncbi.nlm. nih.gov/books/NBK1504/

Ren, J., Aika, Y., Heizmann, C., and Kosaka, T. (1992). Quantitative analysis of neurons and glial cells in the rat somatosensory cortex, with special reference to GABAergic neurons and parvalbumin-containing neurons. Exp. Brain Res. 92, 1-14. doi: 10.1007/BF00230378

Romani, C., Palermo, L., MacDonald, A., Limback, E., Hall, S. K., and Geberhiwot, T. (2017). The impact of phenylalanine levels on cognitive outcomes in adults with phenylketonuria: effects across tasks and developmental stages. Neuropsychology 31:242. doi: 10.1037/neu0000336

Sadasivudu, B., and Lajtha, A. (1970). Metabolism of amino acids in incubated slices of mouse brain. J. Neurochem. 17, 1299-1311. doi: 10.1111/j.1471-4159. 1970.tb03379.x

Schindeler, S., Ghosh-Jerath, S., Thompson, S., Rocca, A., Joy, P., Kemp, A., et al. (2007). The effects of large neutral amino acid supplements in PKU: an MRS and neuropsychological study. Mol. Genet. Metabol. 91, 48-54. doi: 10.1016/j. ymgme.2007.02.002

Segawa, H., Fukasawa, Y., Miyamoto, K., Takeda, E., Endou, H., and Kanai, Y. (1999). Identification and functional characterization of a $\mathrm{Na}+$-independent 
neutral amino acid transporter with broad substrate selectivity. J. Biol. Chem. 274, 19745-19751. doi: 10.1074/jbc.274.28.19745

Setou, M., Hayasaka, T., and Yao, I. (2004). Axonal transport versus dendritic transport. J. Neurobiol. 58, 201-206. doi: 10.1002/neu.10324

Shank, R. P., and Campbell, G. L. (1984). Amino acid uptake, content, and metabolism by neuronal and glial enriched cellular fractions from mouse cerebellum. J. Neurosci. 4, 58-69. doi: 10.1523/JNEUROSCI.04-01-00058.1984

Smith, Q. R., Johanson, C. E., and Woodbury, D. M. (1981). Uptake of 36Cl and $22 \mathrm{Na}$ by the brain-cerebrospinal fluid system: comparison of the permeability of the blood-brain and blood-cerebrospinal fluid barriers. J. Neurochem. 37, 117-124. doi: 10.1111/j.1471-4159.1981.tb05298.x

Smith, Q. R., Momma, S., Aoyagi, M., and Rapoport, S. I. (1987). Kinetics of neutral amino acid transport across the blood-brain barrier. J. Neurochem. 49, 1651-1658. doi: 10.1111/j.1471-4159.1987.tb01039.x

Smith, Q. R., and Takasato, Y. (1986). Kinetics of amino acid transport at the bloodbrain barrier studied using an in situ brain perfusion technique. Ann. N. Y. Acad. Sci. 481, 186-201. doi: 10.1111/j.1749-6632.1986.tb27150.x

Sperringer, J. E., Addington, A., and Hutson, S. M. (2017). Branched-chain amino acids and brain metabolism. Neurochem. Res. 42, 1697-1709. doi: 10.1007/ s11064-017-2261-5

Suraweera, A., Münch, C., Hanssum, A., and Bertolotti, A. (2012). Failure of amino acid homeostasis causes cell death following proteasome inhibition. Mol. Cell 48, 242-253. doi: 10.1016/j.molcel.2012.08.003

Syková, E., Voøíšek, I., Antonova, T., Mazel, T., Meyer-Luehmann, M., Jucker, M., et al. (2005). Changes in extracellular space size and geometry in APP23 transgenic mice: a model of alzheimer's disease. Proc. Natl. Acad. Sci. U.S.A. 102, 479-484. doi: 10.1073/pnas.0408235102

Takanaga, H., Mackenzie, B., Peng, J.-B., and Hediger, M. A. (2005). Characterization of a branched-chain amino-acid transporter SBAT1 (SLC6A15) that is expressed in human brain. Biochem. Biophys. Res. Commun. 337, 892-900. doi: 10.1016/j.bbrc.2005.09.128

Taslimifar, M., Buoso, S., Verrey, F., and Kurtcuoglu, V. (2018). Functional polarity of microvascular brain endothelial cells supported by neurovascular unit computational model of large neutral amino acid homeostasis. Front. Physiol. 9:171. doi: 10.3389/fphys.2018.00171

Tilgmann, C., Melen, K., Lundström, K., Jalanko, A., Julkunen, I., Kalkkinen, N., et al. (1992). Expression of recombinant soluble and membrane-bound catechol O-methyltransferase in eukaryotic cells and identification of the respective enzymes in rat brain. Eur. J. Biochem. 207, 813-821. doi: 10.1111/j.1432-1033. 1992.tb17112.x van Spronsen, F. J., de Groot, M. J., Hoeksma, M., Reijngoud, D.-J., and van Rijn, M. (2010). Large neutral amino acids in the treatment of PKU: from theory to practice. J. Inher. Metab. Dis. 33, 671-676. doi: 10.1007/s10545-0109216-1

van Vliet, D., Bruinenberg, V. M., Mazzola, P. N., van Faassen, M. H., de Blaauw, P., Kema, I. P., et al. (2015). Large neutral amino acid supplementation exerts its effect through three synergistic mechanisms: proof of principle in phenylketonuria mice. PLoS One 10:e0143833. doi: 10.1371/journal.pone. 0143833

van Vliet, D., Bruinenberg, V. M., Mazzola, P. N., van Faassen, M. H., de Blaauw, P., Pascucci, T., et al. (2016). Therapeutic brain modulation with targeted large neutral amino acid supplements in the Pah-enu2 phenylketonuria mouse model. Am. J. Clin. Nutr. 104, 1292-1300. doi: 10.3945/ajcn.116.135996

Verrey, F. (2003). System L: heteromeric exchangers of large, neutral amino acids involved in directional transport. Pflügers Archiv. 445, 529-533. doi: 10.1007/ s00424-002-0973-z

Viau, K. S., Wengreen, H. J., Ernst, S. L., Cantor, N. L., Furtado, L. V., and Longo, N. (2011). Correlation of age-specific phenylalanine levels with intellectual outcome in patients with phenylketonuria. J. Inher. Metab. Dis. 34, 963-971. doi: 10.1007/s10545-011-9329-1

Yudkoff, M. (2017). Interactions in the metabolism of glutamate and the branchedchain amino acids and ketoacids in the CNS. Neurochem. Res. 42, 10-18. doi: 10.1007/s11064-016-2057-z

Yudkoff, M., Daikhin, Y., Grunstein, L., Nissim, I., Stern, J., Pleasure, D., et al. (1996a). Astrocyte leucine metabolism: significance of branched-chain amino acid transamination. J. Neurochem. 66, 378-385.

Yudkoff, M., Daikhin, Y., Nelson, D., Nissim, I., and Erecińska, M. (1996b). Neuronal metabolism of branched-chain amino acids: flux through the aminotransferase pathway in synaptosomes. J. Neurochem. 66, 2136-2145.

Conflict of Interest Statement: The authors declare that the research was conducted in the absence of any commercial or financial relationships that could be construed as a potential conflict of interest.

Copyright (c) 2019 Taslimifar, Buoso, Verrey and Kurtcuoglu. This is an open-access article distributed under the terms of the Creative Commons Attribution License (CC BY). The use, distribution or reproduction in other forums is permitted, provided the original author(s) and the copyright owner(s) are credited and that the original publication in this journal is cited, in accordance with accepted academic practice. No use, distribution or reproduction is permitted which does not comply with these terms. 